\title{
Four pillars of the S-phase checkpoint
}

\author{
Lee $\mathrm{Zou}^{1}$ \\ Massachusetts General Hospital Cancer Center, Harvard Medical School, Charlestown, Massachusetts 02129, USA; Department \\ of Pathology, Massachusetts General Hospital, Harvard Medical School, Boston, Massachusetts 02114, USA
}

The yeast Mec1 kinase is a key regulator of the DNA damage response (DDR). In this issue of Genes \& Development, Kumar and Burgers (pp. 313-321) report that Ddc1, Dpb11, and Dna2 function in concert to activate Mec1 during $S$ phase of the cell cycle. Furthermore, the Tel1 kinase also contributes to the DDR in S phase when Mec1 activation is compromised.

The DNA damage signaling pathway, which is often referred to as the checkpoint, is crucial for genomic stability in all eukaryotes. In human cells, the ATR (ataxia telangiectasia and Rad3-related) kinase is a master regulator of DNA damage signaling (Cimprich and Cortez 2008; Flynn and Zou 2011). In the budding yeast Saccharomyces cerevisiae, Mec1, the ortholog of human ATR, is a key initiator of DNA damage signaling (Melo and Toczyski 2002). During the cell cycle, Mec1 can be activated by a broad spectrum of DNA damage in the G1, S, and G2 phases. In addition, Mec1 can also be activated during $S$ phase by DNA replication stress, such as inhibition of DNA polymerases or depletion of dNTP pools. The activation of Mecl is multistep process that involves (1) recognition of the DNA structures induced by DNA damage and replication stress, (2) recruitment of Mec1 to sites of DNA damage or stressed replication forks, and (3) stimulation of the kinase activity of Mec1 (Flynn and Zou 2011). Two of the Mec1 regulators, Ddc1 and Dpb11, are capable of stimulating the kinase activity of Mec1 in vitro (Majka et al. 2006; Mordes et al. 2008b; Navadgi-Patil and Burgers 2008). Interestingly, Ddc1 and Dpb11 have distinct roles in Mec1 activation during G1 and G2. Ddc1 is the predominant activator of Mec1 in G1, whereas Ddc1 and Dpb11 act redundantly to activate Mec1 in G2 (Navadgi-Patil and Burgers 2009; Zou 2009). Surprisingly, when the functions of Ddc1 and Dpb11 in Mecl activation are both disrupted, Mecl activation still occurs in S phase, suggesting the existence of an additional mechanism for Mecl activation. In the study by Kumar and Burgers (2013) in this issue of Genes \& Development, they identify Dna2 as the third protein that stimulates Mec1 in vitro. Furthermore, they demonstrate

[Keywords: DNA replication; cell cycle checkpoint; 9-1-1; ATR; ATM] ${ }^{1}$ Correspondence

Email zou.lee@mgh.harvard.edu

Article is online at http://www.genesdev.org/cgi/doi/10.1101/gad.213306.113. that during S phase, Dna2 functions in concert with Ddc1 and Dpb11 to activate Mec1 in vivo and that Tell contributes to DNA damage signaling when Mec1 activation is compromised. These findings present a clear picture of how DNA damage signaling is initiated during $S$ phase in budding yeast.

\section{The activation of Mec1 in G1 and G2}

Previous genetic and biochemical studies in yeast and other organisms have revealed a number of regulators of ATR/Mec1 (Cimprich and Cortez 2008; Flynn and Zou 2011). In vertebrates, ATR functions in a complex with its regulatory partner, ATRIP. Similarly, the budding yeast Mec1 and Ddc2 (the ortholog of ATRIP) exist and function as a complex. The ssDNA-binding protein RPA (replication protein A) is an important regulator of ATR/ Mecl in both vertebrates and yeast. RPA-coated ssDNA (RPA-ssDNA) is a common intermediate of DNA repair and is induced by DNA replication stress. Both ATRIP and Ddc2 are able to recognize RPA-ssDNA directly, providing a key mechanism to target ATR and Mecl to sites of DNA damage and stressed replication forks. In addition to RPA, the PCNA-like checkpoint clamp and the RFC-like checkpoint clamp loader are also important to ATR/Mecl activation. In vitro, the human RFClike Rad17-RFC complex is able to recognize the $5^{\prime}$ junctions of ssDNA and dsDNA and recruit the human PCNA-like 9-1-1 (Rad9-Rad1-Hus1) complex onto dsDNA. Similarly, the budding yeast Rad24-RFC complex also recognizes ssDNA-dsDNA junctions and functions as a loader of the yeast 9-1-1 (Ddc1-Mec3-Rad17) complex. Together, RPA, Rad17/Rad24-RFC, and 9-1-1 provide a unique mechanism to sense resected DNA breaks, ssDNA gaps generated by DNA repair, and ssDNA gaps at or behind stressed replication forks (Zou 2007).

In addition to the DNA damage sensors that recognize specific DNA structures, ATR/Mecl activation requires additional factors that stimulate their kinase activities. In vertebrates, a protein called TopBP1 is able to directly stimulate the ATR-ATRIP kinase complex in the absence of DNA and other proteins (Kumagai et al. 2006). Furthermore, TopBP1 is crucial for the phosphorylation of ATR substrates in human cells (Yamane et al. 2003; Liu et al. 2006; Mordes et al. 2008a). Similar to TopBP1, its budding yeast ortholog, Dpb11, also stimulates the Mec1Ddc2 kinase directly (Mordes et al. 2008b; Navadgi-Patil 
and Burgers 2008). Furthermore, Ddc1, a component of the yeast 9-1-1 complex, is able to stimulate Mec1-Ddc2 in vitro under low-salt conditions (Majka et al. 2006). At a physiologically relevant salt concentration, only the yeast 9-1-1 complex but not Ddc1 alone stimulates Mec1-Ddc2. Importantly, only when the 9-1-1 complex is loaded onto DNA structures containing ssDNAdsDNA junctions is it able to stimulate Mec1-Ddc2, suggesting that this function of 9-1-1 is regulated by DNA damage (Majka et al. 2006). These findings in budding yeast suggest that Mecl could be stimulated by multiple factors, raising the question of how Mec1 activation is regulated by these factors in vivo.

While both Ddc1 and Dpb11 are implicated in Mec1 activation genetically, proving them as the Mecl activators in vivo is a challenging task because they are present in complexes with other checkpoint proteins. Furthermore, Dpb11 plays an important role in the initiation of DNA replication at origins, which may affect Mec1 activation indirectly. A critical step toward solving this important problem was made by the Burgers laboratory (Navadgi-Patil and Burgers 2009). In a previous study, Navadgi-Patil and Burgers (2009) carefully analyzed the Mec1 activation domain of Ddc1 and found that an unstructured region near the $\mathrm{C}$ terminus of Ddc1 is both necessary and sufficient for Mecl activation in vitro.
Inspired by a study on the Xenopus TopBP1 (Kumagai et al. 2006), they mutated two aromatic residues (W352 and W544) in this unstructured region and showed that the resulting Ddc1-2W2A mutant is defective for Mec1 activation in vitro. Importantly, although Ddc1-2W2A is unable to stimulate $\mathrm{Mec} 1$ in vitro, it retains the ability to form the 9-1-1 complex and interact with Dpb11. When the $d d c 1-2 W 2 A$ mutant cells were arrested in G1 and treated with the DNA-damaging agent 4-nitroquinoline1-oxide (4-NQO), the phosphorylation of Rad53, a key effector kinase of Mec1, was virtually abolished. This result demonstrates that in G1, Ddc1 is indeed the key activator of Mec1 in vivo (Fig. 1).

Compared with that in G1, the activation of Mec1 in G2 is more complicated. While defective for Rad53 phosphorylation in G1, Ddc1-2W2A is able to support Rad53 phosphorylation in G2. Importantly, the Rad53 phosphorylation in G2 ddc1-2W2A cells is dependent on Dpb11. Dpb11 is known to interact with Ddc1 through the phosphorylated residue T602 (Puddu et al. 2008). The Ddc1-2W2A, T602A triple mutant, which is unable to stimulate Mec1 and interact with Dpb11, is completely defective for Rad53 phosphorylation in G2 cells. Thus, Ddcl contributes to Mecl activation in two distinct ways in G2. First, Ddc1 directly stimulates Mec1 as a component of the 9-1-1 complex. Second, Ddc1 interacts with

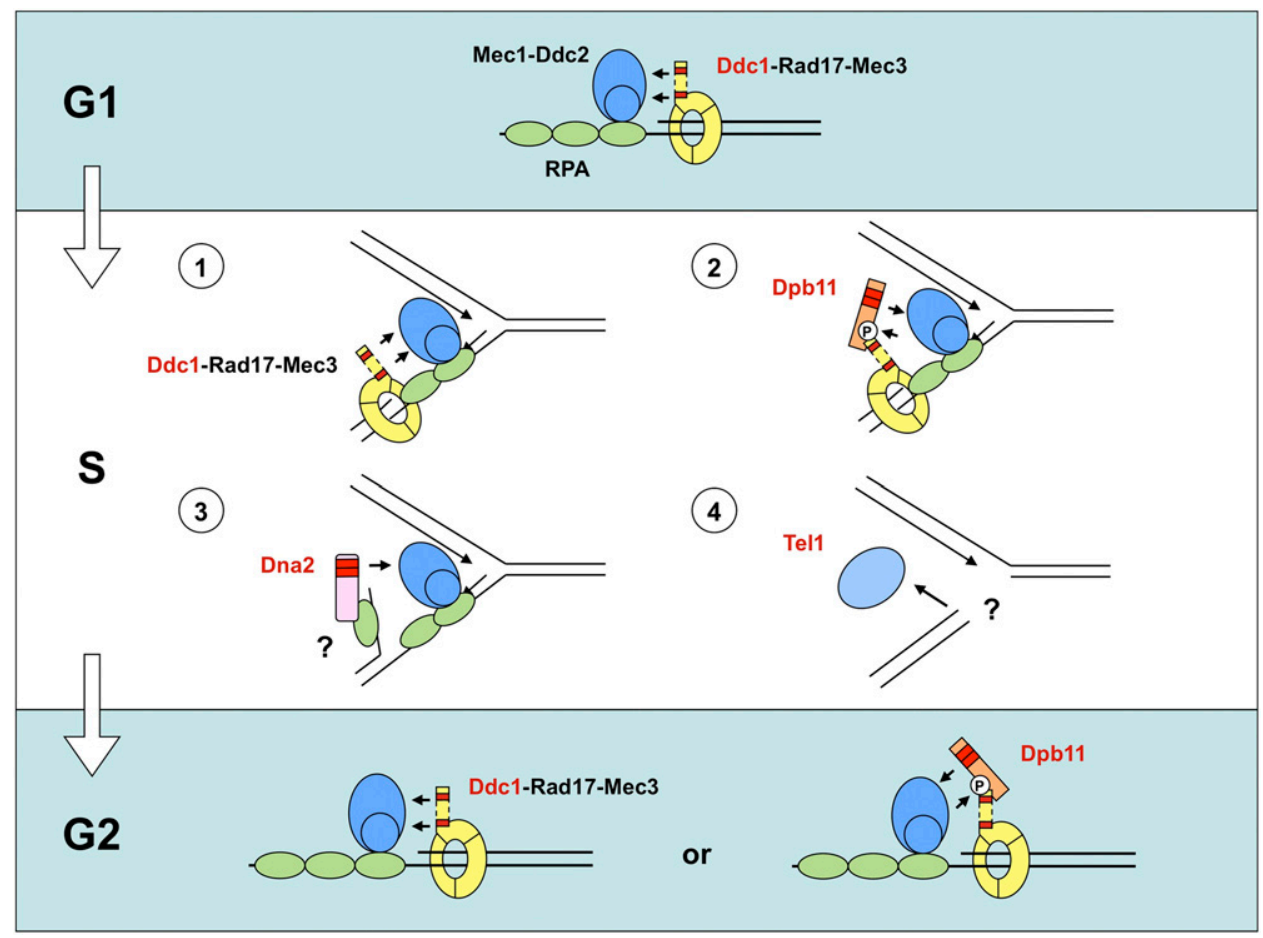

Figure 1. The initiation of DNA damage signaling in budding yeast during the cell cycle. In G1, the Mec1-Ddc2 kinase recruited by RPA-ssDNA is primarily activated by the Ddc1 in the 9-1-1 complex at ssDNA/dsDNA junctions. The aromatic residues of Ddc1 critical for Mec1 activation are depicted as red patches. During S phase, DNA damage signaling can be initiated by four distinct mechanisms: (1) Mec1-Ddc2 is activated by the Ddc1 in the 9-1-1 complex, (2) Mec1-Ddc2 is activated by the Dpb11 associated with Ddc1, (3) Mec1-Ddc2 is activated by Dna2 (the question mark indicates the possibility that Dna2 recognizes RPA-coated 5' flap in this process), and (4) Tell is activated by DNA damage when Mec1 activation is compromised (the question mark indicates that the DNA structures activating Tel1 is still unclear). In G2, Mec1-Ddc2 is activated by either Ddc1 or Dpb11. 
Dpb11 via phosphorylated T602 and enables Dpb11 to stimulate Mec1 (Fig. 1). Similar to Ddc1, Dpb11 stimulates Mec1-Ddc2 using two aromatic residues (W700 and $\mathrm{Y} 735$ ) in an unstructured region (Navadgi-Patil et al. 2011). The Dpb11-W700A, Y735A mutant is proficient for DNA replication but fails to support Rad53 phosphorylation in G2 ddc1-2W2A mutant cells. Together, these results show that Ddc1 and Dpb11 act redundantly in G2 to stimulate Mec1 in vivo (Fig. 1).

Why Mec1 is activated by distinct mechanisms in G1 and G2 is still unclear. Since Dpb11 is needed for the initiation of DNA replication at origins, it may be necessary to prevent Dpb11 from engaging in the DNA damage response (DDR) in G1. Several proteins involved in Mecl activation are phosphorylated by CDK and other cell cycle-regulated kinases in the $S$ and G2 phases. In addition, as exemplified by the resection of DNA breaks, the processing of DNA damage could be differentially regulated in G1 and G2. Regardless of how the mechanisms of Mec1 activation differ between G1 and G2, the activator functions of Ddc1 and Dpb11 are sufficient to explain how Mecl is stimulated in these situations.

\section{The activation of Mec1 in S phase}

During $\mathrm{S}$ phase, the activation of Mecl gets even more complicated than that in G2. The ddc1-2W2A, T602A mutant, which is completely defective for $\operatorname{Rad} 53$ phosphorylation in G2, is still able to support Rad53 phosphorylation in S phase. In fact, Rad53 phosphorylation still occurs in $d d c 1 \Delta$ cells during $S$ phase, suggesting that Mec1 is activated by a Ddc1- and Dpb11-independent mechanism.

To identify the "missing" Mecl activator in S phase, Kumar and Burgers (2013) biochemically tested 20 protein complexes (39 proteins) involved in DNA replication for the ability to stimulate Mec1-Dde2 in vitro. Only one of these proteins, Dna2, directly stimulates Mec1-Ddc2 like Ddc1 and Dpb11. Dna2 is a protein involved in lagging strand maturation during DNA replication (see the next section). Similar to Ddc1 and Dpb11, Dna2 also stimulates Mec1-Ddc2 using two aromatic resides (W128 and Y130) in an unstructured region. When the W128 and Y130 of Dna2 were mutated to alanines, the resulting Dna2-WY-AA mutant failed to stimulate Mec1 in vitro but was still proficient for DNA replication in cells. Surprisingly, however, even in the $d d c 1 \Delta$ dna2-WY-AA double mutant, Rad53 and another Mec1 substrate, Mrc1, were still phosphorylated in S phase, suggesting yet another mechanism to phosphorylate these proteins (Fig. 1).

In addition to $\mathrm{Mec} 1$, budding yeast possesses another PI3K-like protein kinase (PIKK): Tell. Although Tel1 shares sequence homology with the human ATM kinase, it does not play a major role in DNA damage signaling in the presence of Mec1. However, in the absence of Mec1, Tel1 has been shown to phosphorylate some of the Mec1 substrates (Sanchez et al. 1996). Indeed, when Tell was deleted from the dna2-WY-AA mutant, the phosphorylation of Rad53 and Mrcl was partially reduced in S phase (Kumar and Burgers 2013). Furthermore, in the tel1s ddc1s dan2-WY-AA triple mutant, the 4-NQO-induced phosphorylation of Rad53 and Mrcl is virtually abolished in S phase. The hydroxyurea (HU)-induced Rad53 phosphorylation is also eliminated in the triple mutant. Together, these results suggest that the initiation of DNA damage signaling can take place in $S$ phase through four distinct mechanisms: (1) stimulation of Mec1 by Ddc1, (2) stimulation of Mec1 by the Dpb11 associated with Ddc1, (3) stimulation of Mec1 by Dna2, and (4) the function of Tell as a backup kinase of Mec1 (Fig. 1). Only when all four mechanisms are eliminated together is DNA damage signaling abolished in $\mathrm{S}$ phase.

\section{How does Dna2 activate Mec1?}

Dna2 is an essential protein that possesses ssDNA nuclease and $5^{\prime}-3^{\prime}$ DNA helicase activities (Kang et al. 2010). The nuclease activity of Dna2, rather than its helicase activity, is essential for cell survival (Budd et al. 2000; Lee et al. 2000). During DNA replication, the DNA synthesis on the lagging strand is carried out discontinuously as Okazaki fragments. For the synthesis of each Okazaki fragment, a RNA-DNA primer is first generated by the DNA polymerase $\alpha(\operatorname{Pol} \alpha)$-primase, and then extended by the DNA polymerase $\delta$ (Pol $\delta$ ) (Waga and Stillman 1994). When Pol $\delta$ reaches the $5^{\prime}$ end of the previous Okazaki fragment, it displaces the $5^{\prime}$ end and generates a $5^{\prime}$ flap. If the flap is short (1-2 nucleotides [nt]), it is cleaved by the FEN 1 nuclease. If the flap is long (>25 nt) and coated by RPA, it cannot be cut by FEN1 directly. In this situation, the long flap is first cut by Dna2 to generate a short flap, which is subsequently removed by FEN1 (Bae et al. 2001; Rossi and Bambara 2006). Together, Pol $\delta$, FEN1, and Dna2 act in concert to remove the primer of the previous Okazaki fragment, allowing ligation of the two Okazaki fragments by the DNA ligase.

In addition to its role in lagging strand maturation, the nuclease activity of Dna2 is involved in resection of double-stranded DNA breaks (DSBs). In budding yeast, the $5^{\prime}-3^{\prime}$ resection of DSBs is initiated by the MRX (Mre11-Rad50-Xrs2) complex and Sae2 and extended by an Exo1-mediated mechanism or a Dna2-Sgs2-RPAmediated mechanism (Mimitou and Symington 2008; Zhu et al. 2008). During this process, the Sgs2 helicase unwinds DSB ends and generates $5^{\prime}$ flaps, which are recognized by RPA and subsequently removed by Dna2 (Cejka et al. 2010; Niu et al. 2010). In a recent fission yeast study, the endonuclease activity of Dna2 was suggested to prevent accumulation of the "chicken foot" structures at stalled replication forks ( $\mathrm{Hu}$ et al. 2012). Thus, the nuclease activity of Dna2 is implicated in multiple processes important for genomic stability.

Guided by the previous studies on TopBP1, Dpb11, and Ddc1, Kumar and Burgers (2013) postulated that Dna2 might use an unstructured region to activate Mec1. Indeed, the $\mathrm{N}$ terminal region of Dna2 is predicted to be unstructured and does not overlap with the nuclease and helicase domains of Dna2. When tested in vitro, the $\mathrm{N}$-terminal region of Dna2 is sufficient to activate Mec1Ddc2 as efficiently as the full-length Dna2 (Kumar and 
Burgers 2013). Furthermore, similar to the other ATR/ Mec1 activators, Dna2 relies on two aromatic residues (W128 and Y130) in the unstructured region to stimulate Mec1. These results strengthen the hypothesis that a long unstructured region with "bipartite" aromatic residues is a common feature of the ATR/Mec1 activation domains. However, among the known ATR/Mec1 activation domains, the distances between the key aromatic residues vary significantly (Kumar and Burgers 2013). Moreover, several other proteins involved in DNA replication, such as Pif1 and Rrm3, also contain long unstructured regions with aromatic residues but lack the ability to stimulate Mec1. These findings suggest that the ATR/Mecl activation domains may possess additional features important for their functions.

Is the function of Dna2 as a Mec1 activator coupled with its other functions? The temperature-sensitive growth defects of a dna2 mutant are suppressed by FEN1 overexpression (Budd and Campbell 1997), indicating that enhanced FEN1 nuclease function is able to bypass the essential nuclease function of Dna2. However, FEN1 overexpression fails to suppress the growth defects of the tel1s ddc1s dna2-WY-AA triple mutant, suggesting that Dna2-WY-AA is not defective in the nuclease function. Interestingly, although the Mecl activation domain of Dna2 is sufficient to stimulate Mec1-Ddc2 in vitro, its role in $\mathrm{Mecl}$ activation in vivo is restricted to $\mathrm{S}$ phase. When the Mec1 activation domain of Dna2 was fused with Ddc1, it substituted the Mec1 activation domain of Ddc1 and supported Rad53 phosphorylation in G1 (Kumar and Burgers 2013). These results clearly demonstrate that the Mec1 activation domain of Dna2 cannot function alone in vivo. The function of Dna2 as a Mec1 activator requires not only the Mecl activation domain, but also the ability of Dna2 to localize to replication forks and/or sites of DNA damage.

\section{Is Dna2 a sensor of DNA damage at replication forks?}

To understand how Dna2 stimulates Mec1 in S phase, it is important to determine how Dna2 is localized to replication forks and/or sites of DNA damage. Although Dna2 acts in concert with Ddc1 and Dpb11 to stimulate Mec1 in $S$ phase, the functions of these Mecl activators are not identical. For example, the $d d c 1 \Delta$ mutant is partially defective in Rad9 and Rad53 phosphorylation during $S$ phase, but the $\operatorname{dna2}-W Y-A A$ mutant is not. Furthermore, the effects of Dna2-WY-AA on Rad53 and Mrc1 phosphorylation are most evident in the absence of Ddc1 and Tel1, suggesting that Dna2 may primarily function as a backup activator of Mecl when the other mechanisms fail. As mentioned above, 9-1-1 is loaded onto dsDNA by the Rad24-RFC complex at ssDNA-dsDNA junctions. This DNA structural specificity of 9-1-1 provides the critical DNA damage regulation to Ddc1 and Dpb11. If the DNA damage regulation of Dna2 is distinct from Ddc1 and Dpb11, then how is Dna2 regulated?

Dna2 may recognize specific DNA structures at replication forks that are induced by DNA damage or replication stress. Dna2 is known to act on long 5' flaps during lagging strand maturation. In budding yeast, deletion of the Pif1 helicase suppresses the lethality of the dna2s mutant, implicating Pif1 in the generation of the long 5' flaps that are processed by Dna2 (Ryu et al. 2004; Budd et al. 2006). When DNA polymerases are impeded on the lagging strand, the $5^{\prime}$ ends of the previous Okazaki fragments may be persistently exposed. These $5^{\prime}$ ends may be initially recognized by the Rad24-RFC and 9-1-1 complexes. However, in the absence of 9-1-1, Pif1 may gain increased access to these $5^{\prime}$ ends and convert them into long 5 ' flaps, which would facilitate the accumulation of Dna2 at the stressed forks and the Dna2-mediated Mec1 activation (Fig. 1).

Pif1 may not be the only way to generate long 5' flaps in $S$ phase. The contribution of Tel1 to Rad53 phosphorylation in $S$ phase indicates that DSBs are generated during the response to HU and 4-NQO. Long 5' flaps may be present at some of the DSBs generated at collapsed replication forks. Some of the DSBs generated at collapsed forks may be recognized by different resection factors (Cejka et al. 2010; Niu et al. 2010). Furthermore, if the "chicken foot" structures are formed at stalled replication forks, they may present DSB-like dsDNA ends. These DSBs or related structures, once engaged by the Dna2-Sgs2-RPA pathway, may provide a mechanism to recruit both Dna2 and Mec1-Ddc2, allowing Dna2 to stimulate Mec1.

In addition to long $5^{\prime}$ flaps, Dna2 may directly recognize other forms of ssDNA, such as ssDNA gaps and 3' flaps. Like ATRIP, Dna2 is known to interact with RPA through multiple contacts (Bae et al. 2003; Zou and Elledge 2003; Namiki and Zou 2006). During lagging strand maturation and DSB resection, RPA plays an important role in specifying and stimulating Dna2's nuclease activity toward 5' flaps (Kao et al. 2004; MasudaSasa et al. 2008; Cejka et al. 2010; Niu et al. 2010). The ability of Dna2 to bind RPA may enable it to recognize different forms of ssDNA but limit its nuclease activity to 5 ' flaps. If RPA-ssDNA is directly recognized by both Mec1-Ddc2 and Dna2, it may serve as a platform for Dna2 to stimulate $\mathrm{Mec} 1$.

Even if Dna2 does not recognize stress-induced DNA structures at replication forks, it may travel with the forks through its interactions with other replication proteins. In addition to FEN1 and RPA, Dna2 is known to interact with several other replisome components, such as Pol $\delta$, Pol $\alpha$-primase, and Ctf4 (Budd et al. 2005). The ability of Dna2 to travel with the forks may poise it for Mec1 activation during DNA synthesis, resembling the involvements of the replisome components Mrc1, Tof1, and Csm3 in DNA damage signaling.

\section{Why is the checkpoint activated by four distinct mechanisms in $\mathrm{S}$ phase?}

The discovery of four distinct mechanisms for checkpoint activation in $S$ phase raises an important question as to why such an extraordinary functional redundancy is necessary. In budding yeast, Mecl is essential for cell survival unless the dNTP pools are increased by Sml1 
deletion (Zhao et al. 1998). Even in the sml14 background, deletion of both Mec1 and Tell leads to severe growth defects (Myung et al. 2001; Craven et al. 2002). Similar to the sml1s mec1s tel1s mutant, the sml1s tel1s ddc1s dna2 WY-AA quadruple mutant displays severe growth defects (Kumar and Burgers 2013). The poor growth of the quadruple mutant is in marked contrast to the normal growth of the $d d c 1 \Delta$ mutant, which is defective for Mec1 activation in G1 and G2. These results strongly argue that the ability to initiate DNA damage signaling in $\mathrm{S}$ phase is critical to cell survival.

Indeed, even during an unperturbed $S$ phase, Ddc2 and RPA are transiently phosphorylated by Mec1 (Brush et al. 1996; Paciotti et al. 2000), suggesting that Mec1 is elicited by intrinsic stress during DNA replication. When Mecl's function is compromised in the absence of extrinsic stress, chromosomes fail to complete replication with normal kinetics in $S$ phase and are increasingly fragmented in specific replication slow zones (RSZs) and at compromised early origins (CEOs) (Cha and Kleckner 2002; Raveendranathan et al. 2006). Consistent with these pre-

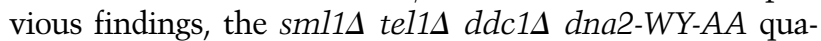
druple mutant, which lacks all four mechanisms to initiate DNA damage signaling in S phase, fails to complete DNA replication efficiently even in the absence of $\mathrm{HU}$ (Kumar and Burgers 2013). In contrast to the quadruple mutant, the sml1s tel1s ddc1s triple mutant that contains wildtype Dna2 progresses through S phase without an obvious delay, suggesting that Dna2 alone is sufficient to support the critical function of Mec1 in DNA replication. Furthermore, the sml1s tel1s ddc1s triple mutant progresses through $S$ phase much more efficiently than the sml1s tel1s ddc1s dna2-WY-AA quadruple mutant in the presence of $\mathrm{HU}$, showing that even one of the four mechanisms to initiate DNA damage signaling (Dna2-mediated Mecl activation) is able to cope with HU-induced replication stress. Whether the different mechanisms to initiate DNA damage signaling in $S$ phase are important for the response to different cellular stresses remains to be tested. The multiple mechanisms to initiate DNA damage signaling in S phase may not only provide a safety net for DNA replication, but also afford cells the ability to cope with a wide range of cellular stress.

\section{Further questions about Dna2 and ATR activation}

The discovery of Dna2 as an S-phase-specific activator of Mec1 in budding yeast raises a number of interesting questions for future studies. One of these questions is how the function of Dna2 in Mec1 activation is restricted to $\mathrm{S}$ phase. Interestingly, in budding yeast, the bulk of Dna2 is localized to telomeres in G1 and G2 (Choe et al. 2002). Furthermore, Dna2 is a substrate of CDK (Chen et al. 2011). In fission yeast, Cds1, an effector kinase of ATR/Rad3, phosphorylates Dna2 and promotes its retention at stalled replication forks (Hu et al. 2012). In addition to the regulation of Dna2 itself, the function of Dna2 in Mec1 activation may be controlled by specific DNA structures during $S$ phase. In the presence of DNA damage or replication stress, the DNA structures that regulate Dna2 could be generated by proteins that function at replication forks or by processing factors that are activated during $\mathrm{S}$ phase. These regulatory mechanisms of Dna2 may define a cell cycle window in which Dna2 is able to activate Mec1 efficiently.

Another question about the role of Dna2 in Mec1 activation is whether it has a unique specificity for DNA damage. Although the functional redundancy among Ddc1, Dpb11, and Dna2 is clear, these Mec1 activators are likely regulated by distinct mechanisms. Elucidating how Dna2 activates Mec1 on DNA and how the role of Dna2 is different from those of Ddc1 and Dpb11 will help us understand how these Mecl activators function in concert during $S$ phase. The potential function of Dna2 in Mecl activation outside of $\mathrm{S}$ phase is also worth exploring. The Dna2-Sgs2-RPA pathway may actively engage in DSB resection in both S and G2 phases. It would be interesting to determine whether Dna2 is able to stimulate Mec1 in the context of resected DSBs in G2.

The study by Kumar and Burgers (2013) also raises the important question of whether the vertebrate ATR kinase can be stimulated by multiple activators. Whether the human 9-1-1 complex and Dna2 can stimulate the ATR-ATRIP kinase is still unknown. In asynchronously growing human cells, ATR phosphorylates its effector kinase, Chk1, in response to DNA damage in a TopBP1dependent manner (Yamane et al. 2003; Liu et al. 2006). In Xenopus egg extracts, the phosphorylation of Chk1 by ATR in response to replication inhibition and DSBs is also dependent on TopBP1 (Hashimoto et al. 2006; Kumagai et al. 2006). These findings suggest that TopBP1 may be the predominant activator of ATR in vertebrates. Interestingly, in human cells, the levels of TopBP1 are low outside of $S$ phase (Yamane et al. 2002). However, ATR-dependent $\mathrm{H} 2 \mathrm{AX}$ phosphorylation was detected in nonproliferating cells (O'Driscoll et al. 2003), and ATR-mediated checkpoint responses were observed in G2 (Stiff et al. 2008). In addition, the DNA damage-induced ATR autophosphorylation at T1989 is independent of TopBP1 (Liu et al. 2011). Although it is still unclear whether these ATR-mediated events are dependent on the stimulation of ATR kinase activity, they raise the possibility that ATR may perform some of its functions independently of TopBP1.

The finding of multiple mechanisms to initiate DNA damage signaling in $\mathrm{S}$ phase highlights the importance of the DNA damage signaling pathway in DNA replication. In vertebrates, although it is still unclear whether ATR can be activated by multiple mechanisms, the functional redundancy among the different PIKKs is evident. Unlike the yeast Tell, the human ATM kinase plays a crucial role in the response to DSBs throughout the cell cycle. ATM not only phosphorylates many important substrates directly, it also promotes the activation of ATR by DSBs in the $S$ and G2 phases (Jazayeri et al. 2006; Myers and Cortez 2006). In Xenopus extracts, both ATM and ATR are important for preventing accumulation of DSBs during DNA replication (Trenz et al. 2006). In human cells, DNA-PKcs, a PIKK that is absent in yeast, also contributes to DNA damage signaling. Furthermore, recent studies have implicated DNA-PKcs in the 
replication stress response (Yajima et al. 2009; Liu et al. 2012). Thus, although ATR, ATM, and DNA-PKcs each have unique functions, they also work as a group to initiate DNA damage signaling in $S$ phase. To better understand how DNA damage signaling is initiated during $S$ phase in human cells, it would be important to elucidate how ATR, ATM, and DNA-PKcs are activated and how they act in concert in $S$ phase. In addition, it will be a formidable challenge for future studies to reveal how the DNA damage signaling pathway protects the genome during DNA replication under different cellular stresses.

\section{Acknowledgments}

I apologize to the colleagues whose work is not cited due to space constraints. Work in my laboratory is supported by grants from NIH (GM076388) and the MGH/NCI Proton Program. I am a Jim and Ann Orr MGH Research Scholar and a Scholar of the Ellison Medical Foundation.

\section{References}

Bae SH, Bae KH, Kim JA, Seo YS. 2001. RPA governs endonuclease switching during processing of Okazaki fragments in eukaryotes. Nature 412: 456-461.

Bae KH, Kim HS, Bae SH, Kang HY, Brill S, Seo YS. 2003. Bimodal interaction between replication-protein A and Dna2 is critical for Dna2 function both in vivo and in vitro. Nucleic Acids Res 31: 3006-3015.

Brush GS, Morrow DM, Hieter P, Kelly TJ. 1996. The ATM homologue MEC1 is required for phosphorylation of replication protein A in yeast. Proc Natl Acad Sci 93: 15075-15080.

Budd ME, Campbell JL. 1997. A yeast replicative helicase, Dna2 helicase, interacts with yeast FEN-1 nuclease in carrying out its essential function. Mol Cell Biol 17: 2136-2142.

Budd ME, Choe W, Campbell JL. 2000. The nuclease activity of the yeast DNA2 protein, which is related to the RecBlike nucleases, is essential in vivo. I Biol Chem 275: 1651816529.

Budd ME, Tong AH, Polaczek P, Peng X, Boone C, Campbell JL. 2005. A network of multi-tasking proteins at the DNA replication fork preserves genome stability. PLoS Genet 1: e61.

Budd ME, Reis CC, Smith S, Myung K, Campbell JL. 2006. Evidence suggesting that Pifl helicase functions in DNA replication with the Dna2 helicase/nuclease and DNA polymerase $\delta$. Mol Cell Biol 26: 2490-2500.

Cejka P, Cannavo E, Polaczek P, Masuda-Sasa T, Pokharel S, Campbell JL, Kowalczykowski SC. 2010. DNA end resection by Dna2-Sgs1-RPA and its stimulation by Top3-Rmil and Mre11-Rad50-Xrs2. Nature 467: 112-116.

Cha RS, Kleckner N. 2002. ATR homolog Mec1 promotes fork progression, thus averting breaks in replication slow zones. Science 297: 602-606.

Chen X, Niu H, Chung WH, Zhu Z, Papusha A, Shim EY, Lee SE, Sung P, Ira G. 2011. Cell cycle regulation of DNA doublestrand break end resection by Cdk1-dependent Dna2 phosphorylation. Nat Struct Mol Biol 18: 1015-1019.

Choe W, Budd M, Imamura O, Hoopes L, Campbell JL. 2002. Dynamic localization of an Okazaki fragment processing protein suggests a novel role in telomere replication. Mol Cell Biol 22: 4202-4217.

Cimprich KA, Cortez D. 2008. ATR: An essential regulator of genome integrity. Nat Rev Mol Cell Biol 9: 616-627.
Craven RJ, Greenwell PW, Dominska M, Petes TD. 2002. Regulation of genome stability by TEL1 and MEC1, yeast homologs of the mammalian ATM and ATR genes. Genetics 161: 493-507.

Flynn RL, Zou L. 2011. ATR: A master conductor of cellular responses to DNA replication stress. Trends Biochem Sci 36: 133-140.

Hashimoto Y, Tsujimura T, Sugino A, Takisawa H. 2006. The phosphorylated C-terminal domain of Xenopus Cut5 directly mediates ATR-dependent activation of Chk1. Genes Cells 11: 993-1007.

Hu J, Sun L, Shen F, Chen Y, Hua Y, Liu Y, Zhang M, Hu Y, Wang Q, Xu W, et al. 2012. The intra-S phase checkpoint targets Dna2 to prevent stalled replication forks from reversing. Cell 149: 1221-1232.

Jazayeri A, Falck J, Lukas C, Bartek J, Smith GC, Lukas J, Jackson SP. 2006. ATM- and cell cycle-dependent regulation of ATR in response to DNA double-strand breaks. Nat Cell Biol 8: 37-45.

Kang YH, Lee CH, Seo YS. 2010. Dna2 on the road to Okazaki fragment processing and genome stability in eukaryotes. Crit Rev Biochem Mol Biol 45: 71-96.

Kao HI, Veeraraghavan J, Polaczek P, Campbell JL, Bambara RA. 2004. On the roles of Saccharomyces cerevisiae Dna2p and Flap endonuclease 1 in Okazaki fragment processing. I Biol Chem 279: 15014-15024.

Kumagai A, Lee J, Yoo HY, Dunphy WG. 2006. TopBP1 activates the ATR-ATRIP complex. Cell 124: 943-955.

Kumar S, Burgers PM. 2013. Lagging strand maturation factor Dna2 is a component of the replication checkpoint initiation machinery. Genes Dev (this issue). doi: 10.1101/gad.204750.112.

Lee KH, Kim DW, Bae SH, Kim JA, Ryu GH, Kwon YN, Kim KA, Koo HS, Seo YS. 2000. The endonuclease activity of the yeast Dna2 enzyme is essential in vivo. Nucleic Acids Res 28: 2873-2881.

Liu S, Bekker-Jensen S, Mailand N, Lukas C, Bartek J, Lukas J. 2006. Claspin operates downstream of TopBP1 to direct ATR signaling towards Chk1 activation. Mol Cell Biol 26: 60566064.

Liu S, Shiotani B, Lahiri M, Marechal A, Tse A, Leung CC, Glover JN, Yang XH, Zou L. 2011. ATR autophosphorylation as a molecular switch for checkpoint activation. Mol Cell 43: 192-202.

Liu S, Opiyo SO, Manthey K, Glanzer JG, Ashley AK, Amerin C, Troksa K, Shrivastav M, Nickoloff JA, Oakley GG. 2012. Distinct roles for DNA-PK, ATM and ATR in RPA phosphorylation and checkpoint activation in response to replication stress. Nucleic Acids Res 40: 10780-10794.

Majka J, Niedziela-Majka A, Burgers PM. 2006. The checkpoint clamp activates Mec1 kinase during initiation of the DNA damage checkpoint. Mol Cell 24: 891-901.

Masuda-Sasa T, Polaczek P, Peng XP, Chen L, Campbell JL. 2008. Processing of G4 DNA by Dna2 helicase/nuclease and replication protein A (RPA) provides insights into the mechanism of Dna2/RPA substrate recognition. J Biol Chem 283: 2435924373.

Melo J, Toczyski D. 2002. A unified view of the DNA-damage checkpoint. Curr Opin Cell Biol 14: 237-245.

Mimitou EP, Symington LS. 2008. Sae2, Exo1 and Sgs1 collaborate in DNA double-strand break processing. Nature 455: 770-774

Mordes DA, Glick GG, Zhao R, Cortez D. 2008a. TopBP1 activates ATR through ATRIP and a PIKK regulatory domain. Genes Dev 22: 1478-1489.

Mordes DA, Nam EA, Cortez D. 2008b. Dpb11 activates the Mec1-Ddc2 complex. Proc Natl Acad Sci 105: 18730-18734. 
Myers JS, Cortez D. 2006. Rapid activation of ATR by ionizing radiation requires ATM and Mre11. J Biol Chem 281: 93469350.

Myung K, Datta A, Kolodner RD. 2001. Suppression of spontaneous chromosomal rearrangements by $\mathrm{S}$ phase checkpoint functions in Saccharomyces cerevisiae. Cell 104: 397408.

Namiki Y, Zou L. 2006. ATRIP associates with replication protein A-coated ssDNA through multiple interactions. Proc Natl Acad Sci 103: 580-585.

Navadgi-Patil VM, Burgers PM. 2008. Yeast DNA replication protein Dpb11 activates the Mec1/ATR checkpoint kinase. I Biol Chem 283: 35853-35859.

Navadgi-Patil VM, Burgers PM. 2009. The unstructured C-terminal tail of the 9-1-1 clamp subunit Ddc1 activates Mec1/ATR via two distinct mechanisms. Mol Cell 36: 743-753.

Navadgi-Patil VM, Kumar S, Burgers PM. 2011. The unstructured C-terminal tail of yeast Dpb11 (human TopBP1) protein is dispensable for DNA replication and the $S$ phase checkpoint but required for the G2/M checkpoint. I Biol Chem 286: 40999-41007.

Niu H, Chung WH, Zhu Z, Kwon Y, Zhao W, Chi P, Prakash R, Seong C, Liu D, Lu L, et al. 2010. Mechanism of the ATPdependent DNA end-resection machinery from Saccharomyces cerevisiae. Nature 467: 108-111.

O'Driscoll M, Ruiz-Perez VL, Woods CG, Jeggo PA, Goodship JA. 2003. A splicing mutation affecting expression of ataxiatelangiectasia and Rad3-related protein (ATR) results in Seckel syndrome. Nat Genet 33: 497-501.

Paciotti V, Clerici M, Lucchini G, Longhese MP. 2000. The checkpoint protein $\mathrm{Ddc} 2$, functionally related to $S$. pombe Rad26, interacts with Mec1 and is regulated by Mec1dependent phosphorylation in budding yeast. Genes Dev 14: 2046-2059.

Puddu F, Granata M, Di Nola L, Balestrini A, Piergiovanni G, Lazzaro F, Giannattasio M, Plevani P, Muzi-Falconi M. 2008. Phosphorylation of the budding yeast 9-1-1 complex is required for Dpb11 function in the full activation of the UV-induced DNA damage checkpoint. Mol Cell Biol 28: 4782-4793.

Raveendranathan $M$, Chattopadhyay S, Bolon YT, Haworth J, Clarke DJ, Bielinsky AK. 2006. Genome-wide replication profiles of S-phase checkpoint mutants reveal fragile sites in yeast. $E M B O$ J 25: 3627-3639.

Rossi ML, Bambara RA. 2006. Reconstituted Okazaki fragment processing indicates two pathways of primer removal. J Biol Chem 281: 26051-26061.

Ryu GH, Tanaka H, Kim DH, Kim JH, Bae SH, Kwon YN, Rhee JS, MacNeill SA, Seo YS. 2004. Genetic and biochemical analyses of Pfh1 DNA helicase function in fission yeast. Nucleic Acids Res 32: 4205-4216.

Sanchez Y, Desany BA, Jones WJ, Liu Q, Wang B, Elledge SJ. 1996. Regulation of RAD53 by the ATM-like kinases MEC1 and TEL1 in yeast cell cycle checkpoint pathways. Science 271: $357-360$.

Stiff T, Cerosaletti K, Concannon P, O'Driscoll M, Jeggo PA. 2008. Replication independent ATR signalling leads to G2/M arrest requiring Nbs1, 53BP1 and MDC1. Hum Mol Genet 17: 3247-3253.

Trenz K, Smith E, Smith S, Costanzo V. 2006. ATM and ATR promote Mre11 dependent restart of collapsed replication forks and prevent accumulation of DNA breaks. EMBO J 25: 1764-1774.

Waga S, Stillman B. 1994. Anatomy of a DNA replication fork revealed by reconstitution of SV40 DNA replication in vitro. Nature 369: 207-212.
Yajima H, Lee KJ, Zhang S, Kobayashi J, Chen BP. 2009. DNA double-strand break formation upon UV-induced replication stress activates ATM and DNA-PKcs kinases. J Mol Biol 385: 800-810.

Yamane K, Wu X, Chen J. 2002. A DNA damage-regulated BRCTcontaining protein, TopBP1, is required for cell survival. Mol Cell Biol 22: 555-566.

Yamane K, Chen J, Kinsella TJ. 2003. Both DNA topoisomerase II-binding protein 1 and BRCA1 regulate the G2-M cell cycle checkpoint. Cancer Res 63: 3049-3053.

Zhao X, Muller EG, Rothstein R. 1998. A suppressor of two essential checkpoint genes identifies a novel protein that negatively affects dNTP pools. Mol Cell 2: 329-340.

Zhu Z, Chung WH, Shim EY, Lee SE, Ira G. 2008. Sgs1 helicase and two nucleases Dna2 and Exo1 resect DNA double-strand break ends. Cell 134: 981-994.

Zou L. 2007. Single- and double-stranded DNA: Building a trigger of ATR-mediated DNA damage response. Genes Dev 21: 879-885.

Zou L. 2009. Checkpoint Mec-tivation comes in many flavors. Mol Cell 36: 734-735.

Zou L, Elledge SJ. 2003. Sensing DNA damage through ATRIP recognition of RPA-ssDNA complexes. Science 300: 15421548. 


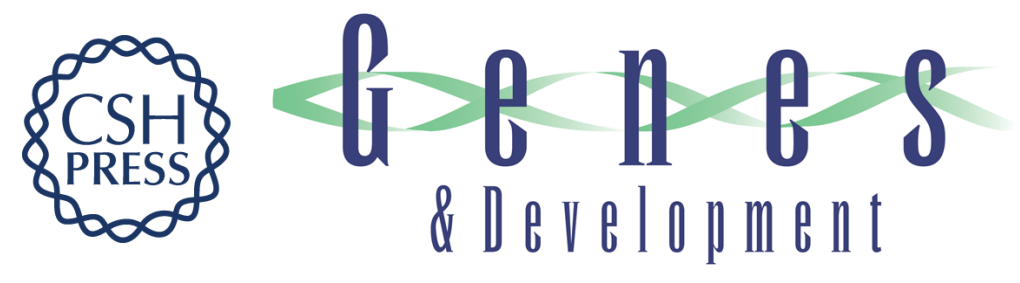

\section{Four pillars of the S-phase checkpoint}

\section{Lee Zou}

Genes Dev. 2013, 27:

Access the most recent version at doi:10.1101/gad.213306.113

\section{Related Content Lagging strand maturation factor Dna2 is a component of the replication checkpoint initiation machinery \\ Sandeep Kumar and Peter M. Burgers \\ Genes Dev. February , 2013 27: 313-321 \\ References This article cites 55 articles, 23 of which can be accessed free at: http://genesdev.cshlp.org/content/27/3/227.full.html\#ref-list-1 \\ Articles cited in: \\ http://genesdev.cshlp.org/content/27/3/227.full.html\#related-urls \\ License \\ Email Alerting \\ Service \\ Receive free email alerts when new articles cite this article - sign up in the box at the top right corner of the article or click here.}

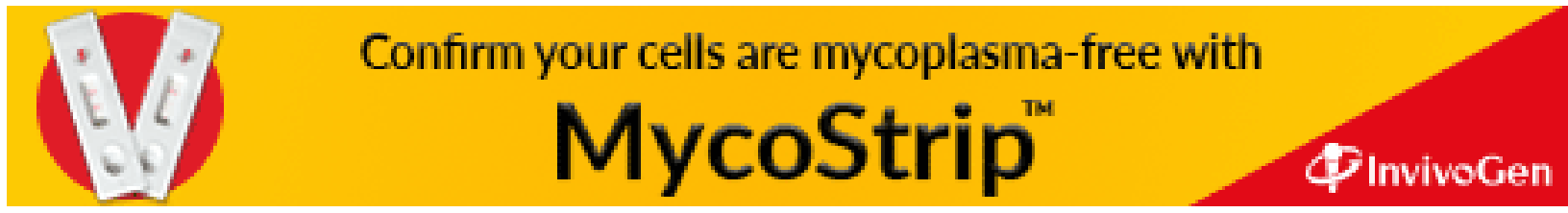

